Taxonomía y Sistemática

\title{
Primeros registros del género Nopachtus (Xenarthra: Cingulata: Glyptodontidae) en Uruguay
}

\author{
First record of the genus Nopachtus (Xenarthra: Cingulata: Glyptodontidae) in Uruguay \\ Martín Zamorano ${ }^{\mathrm{a}, *}$, Pablo Toriño ${ }^{\mathrm{b}}$, Andrés Rinderknecht ${ }^{\mathrm{b}, \mathrm{c}}$ y Daniel A. Perea ${ }^{\mathrm{b}}$ \\ ${ }^{a}$ División Paleontología de Vertebrados, Museo de La Plata, Facultad de Ciencias Naturales y Museo, Universidad Nacional de La Plata, Consejo Nacional de \\ Investigaciones Científicas y Técnicas (CONICET). Paseo del Bosque s/n, 1900, La Plata, Argentina \\ ${ }^{\mathrm{b}}$ Instituto de Ciencias Geológicas, Facultad de Ciencias, Universidad de la República, Iguá 4225, 11.400, Montevideo, Uruguay \\ ${ }^{\mathrm{c}}$ Museo Nacional de Historia Natural, 25 de mayo 582, CC 399, Montevideo, Uruguay
}

Recibido el 30 de marzo de 2015; aceptado el 2 de septiembre de 2015

Disponible en Internet el 9 de febrero de 2016

\begin{abstract}
Resumen
Nopachtus Ameghino es un género de gliptodóntido poco conocido. Cuenta con 2 especies registradas para Argentina: Nopachtus coagmentatus Ameghino, de la formación Brochero (Montehermosense-Chapadmalalense, Plioceno Temprano-Plioceno Tardío) de las sierras de Córdoba/formación El Polvorín (Chapadmalalense Superior, Plioceno) de las sierras Bayas de Olavarría, provincia de Buenos Aires; y Nopachtus cabrerai Zamorano, Scillato-Yané, González-Ruiz y Zurita, de la formación Monte Hermoso (Montehermosense, Plioceno Temprano) del SO de la provincia de Buenos Aires. El conocimiento sobre la presencia de Nopachtus fuera de Argentina se debe a la mención de Toriño y Rinderknecht (2005) de un osteodermo que posee características semejantes a Nopachtus y a Panochthus intermedius Lydekker; posteriormente, Toriño y Perea (2008) retoman esta consideración. En la presente contribución se realiza un estudio de materiales referidos a Nopachtus en Uruguay. Se trata de 2 osteodermos aislados correspondientes a la coraza dorsal: FC-CVF 1825 y FC-CVF 2759, ambos proceden de la formación Camacho (Mioceno Tardío) o formación Raigón (Mioceno Tardío-Pleistoceno Medio) de las barrancas costeras del departamento de San José, Uruguay. Dada la notoria semejanza de estos osteodermos con los de Nopachtus, y por comparaciones realizadas, se asignan al citado género, ampliándose así la distribución geográfica del taxón a Uruguay.

Derechos Reservados ( 2015 Universidad Nacional Autónoma de México, Instituto de Biología. Este es un artículo de acceso abierto distribuido bajo los términos de la Licencia Creative Commons CC BY-NC-ND 4.0.
\end{abstract}

Palabras clave: Gliptodóntido; Osteodermo; Mioceno Tardío; Distribución geográfica

\begin{abstract} Creative Commons CC License BY-NC-ND 4.0.

Keywords: Glyptodontid; Osteoderm; Late Miocene; Geographic distribution

\footnotetext{
* Autor para correspondencia.

Correo electrónico: marzamorano@fcnym.unlp.edu.ar (M. Zamorano).

La revisión por pares es responsabilidad de la Universidad Nacional Autónoma de México.
}

Nopachtus Ameghino is a poorly known genus of glyptodontid. The genus includes 2 species recorded in Argentina: Nopachthus coagmentatus Ameghino, from Brochero Formation (Montehermosian-Chapadmalalian, Early Pliocene-Late Pliocene) of Sierras de Córdoba/El Polvorín Formation (Upper Chapadmalalian, Pliocene) of Sierras Bayas de Olavarría, Buenos Aires province, and Nopachthus cabrerai Zamorano, Scillato-Yané, González-Ruiz and Zurita, from Monte Hermoso Formation (Montehermosian, Early Pliocene) from SO of Buenos Aires province. The knowledge about the presence of Nopachtus outside Argentina, is due to Toriño y Rinderknecht (2005), who mention one osteoderm which possesses similar characters to those of Nopachtus and Panochthus intermedius Lydekker. Later, Toriño y Perea (2008) resume this consideration. In this paper, the materials referred to Nopachtus in Uruguay are studied. There are 2 isolated osteoderms corresponding to the dorsal carapace: FC-CVF 1825 and CF-CVF 2759, both from Camacho Formation (Late Miocene) or Raigón Formation (Late Miocene-Middle Pleistocene) of the coast cliffs of San José Departament, Uruguay. Due to the great similarity of these osteoderms to those of Nopachtus, and according to the comparative analysis conducted, both are assigned to the present genus, thus expanding the geographic distribution of this taxon to Uruguay.

All Rights Reserved (C) 2015 Universidad Nacional Autónoma de México, Instituto de Biología. This is an open access item distributed under the 


\section{Introducción}

Nopachtus Ameghino es un género poco conocido de la familia Glyptodontidae. Está constituido por 2 especies registradas para Argentina: Nopachtus coagmentatus Ameghino - la especie tipo-y Nopachthus cabrerai Zamorano, Scillato-Yané, González-Ruiz y Zurita. Nopachtus coagmentatus fue reconocida por Ameghino (1888), con base en un fragmento lateral de coraza y partes del tubo caudal, hallado en las sierras de la provincia de Córdoba en sedimentos mio-pliocenos («Bocherense», sensu Castellanos, 1942) aflorantes en las cercanías de Villa Cura Brochero; recientemente Zamorano, de los Reyes, Poiré y Scillato-Yané (2015) atribuyen a esta especie osteodermos de la coraza dorsal procedentes de la formación El Polvorín (piso/edad Chapadmalalense Superior, Plioceno) de las sierras Bayas de Olavarría, provincia de Buenos Aires. Nopachtus cabrerai fue descrita por Zamorano, Scillato-Yané, GonzálezRuíz y Zurita (2011), con base en la mitad derecha de una coraza originalmente identificada por Castellanos (1942) como «Nopachthus»(sic) trouessarti (Moreno) (Moreno, 1888) (especie actualmente incluida en el género Phlyctaenopyga Cabrera, ver Zamorano et al., 2011), procedente de la base de las barrancas de Monte Hermoso formación Monte Hermoso (piso/edad Montehermosense, Plioceno Temprano, ver Zamorano, 2013).

De acuerdo con la diagnosis enmendada establecida por Zamorano et al. (2011) y Zamorano (2012a), Nopachtus se caracteriza por ser un gliptodóntido de talla mediana a medianogrande, algo mayor que Phlyctaenopyga, pero menor que Panochthus Burmeister, cuyos osteodermos presentan una figura central conspicua, plana en la región anterior de la coraza y tendiendo a convexa en la posterior - aunque no tanto como en Phlyctaenopyga-, rodeada generalmente por 2 hileras de figuritas periféricas: la primera hilera completa formada por 9 a 12 figuritas, y la segunda no siempre completa, formada por aproximadamente una veintena de figuritas en algunos casos.

Desde la propuesta original de Castellanos (1927) establecida con base en presuntas similitudes en la ornamentación de la coraza, este género ha constituido tradicionalmente una agrupación taxonómica junto a Panochthus (actualmente constituido por 6 especies; Zamorano, 2012a, 2012b; Zamorano, Scillato-Yané y Zurita, 2014) y Propanochthus Castellanos (con una única especie: Propanochthus bullifer Burmeister), primariamente reconocida esta como una subfamilia (Panochthinae, sensu Castellanos, 1927) y luego como una tribu (Panochthini, sensu Simpson, 1945). Esta última adquirió una notable aceptación en las décadas siguientes, siendo incluida en la subfamilia «Hoplophorinae»(e.g., Fernicola, 2008; Hoffstetter, 1958; Paula-Couto, 1979; Scillato-Yané, Carlini, Vizcaíno y OrtizJaureguizar, 1995; Zamorano, 2012a, 2012b; Zamorano et al., 2011; Zamorano y Brandoni, 2013).

No obstante, la monofilia de la tribu «Panochthini»ha comenzado a ser cuestionada en recientes análisis filogenéticos, del mismo modo que también lo ha sido la de la subfamilia «Hoplophorinae»(e.g., Fernicola, 2008; Fernicola y Porpino, 2012; Porpino, Fernicola y Bergqvist, 2010; Zamorano y Brandoni, 2013). De acuerdo con Zamorano (2012a, 2012b) y Zamorano y Brandoni (2013), los «Panochthini» no son un grupo natural, siendo Nopachtus y Propanochthus géneros más emparentados con los representantes de la tribu «Plohophorini», mientras que Panochthus forma un clado junto a Hoplophorus Lund (tribu «Hoplophorini»propuesta por Zamorano, Zurita y Scillato-Yané, 2013). Dado que actualmente la sistemática de los «Hoplophorinae»y sus tribus se encuentra en plena revisión, en el presente trabajo no se sigue el sistema de clasificación tradicional propuesto por Hoffstetter (1958).

En lo referente a la presencia del género Nopachtus fuera de Argentina, Toriño y Rinderknecht (2005) describen sucintamente un osteodermo de coraza dorsal, recolectado en las costas del balneario Kiyú, departamento de San José, Uruguay (fig. 1), al que identifican como perteneciente a un «Panochthini", señalando que posee características que lo asemejan a Nopachtus y a Panochthus intermedius Lydekker. Posteriormente, Toriño y Perea (2008), en el marco de una lista comentada de gliptodóntidos del Terciario de Uruguay, retoman esta consideración, indicando la existencia de más de un osteodermo, y acotando la presunta similitud de los mismos al primero de los géneros mencionados, aunque sin brindar mayor información. En la presente contribución se realiza un estudio detallado de los referidos materiales recolectados en Uruguay, dándose a conocer por primera vez para el país la presencia de osteodermos asignables al género Nopachtus.

\section{Materiales y métodos}

El material descrito se encuentra depositado en la colección paleontológica de la Facultad de Ciencias, Montevideo, Uruguay, y fue comparado con ejemplares presentes en las colecciones de las instituciones que se detallan a continuación. Abreviaturas: FC-CVF, colección de vertebrados fósiles. Facultad de Ciencias, Universidad de la República. Montevideo, Uruguay; MACN-Pv, Colección Nacional de Paleovertebrados, Museo Argentino de Ciencias Naturales «Bernardino Rivadavia», Ciudad Autónoma de Buenos Aires, Argentina; MLP, División Paleontología Vertebrados, Facultad de Ciencias Naturales y Museo de La Plata, provincia de Buenos Aires, Argentina; Xen-45: colección Cementos Avellaneda, Olavarría, provincia de Buenos Aires, Argentina.

\section{Descripción}

Magnorden Xenarthra Cope, 1889

Orden Cingulata Illiger, 1811

Suborden Glyptodontia Ameghino, 1889

Superfamilia Glyptodontoidea Gray, 1869

Familia Glyptodontidae Gray, 1869

Género Nopachtus Ameghino, 1888

Sinonimia. Nopachthus. Emmendatio illegitima pro Nopachtus Ameghino, 1888 (ver Castellanos, 1925 y publicaciones posteriores del autor; Mones, 1986: 237; ICZN, 1999: Art. 33).

Especie tipo. Nopachtus coagmentatus Ameghino, 1888; designación original. Piso/edad Montehermosense, Argentina. 


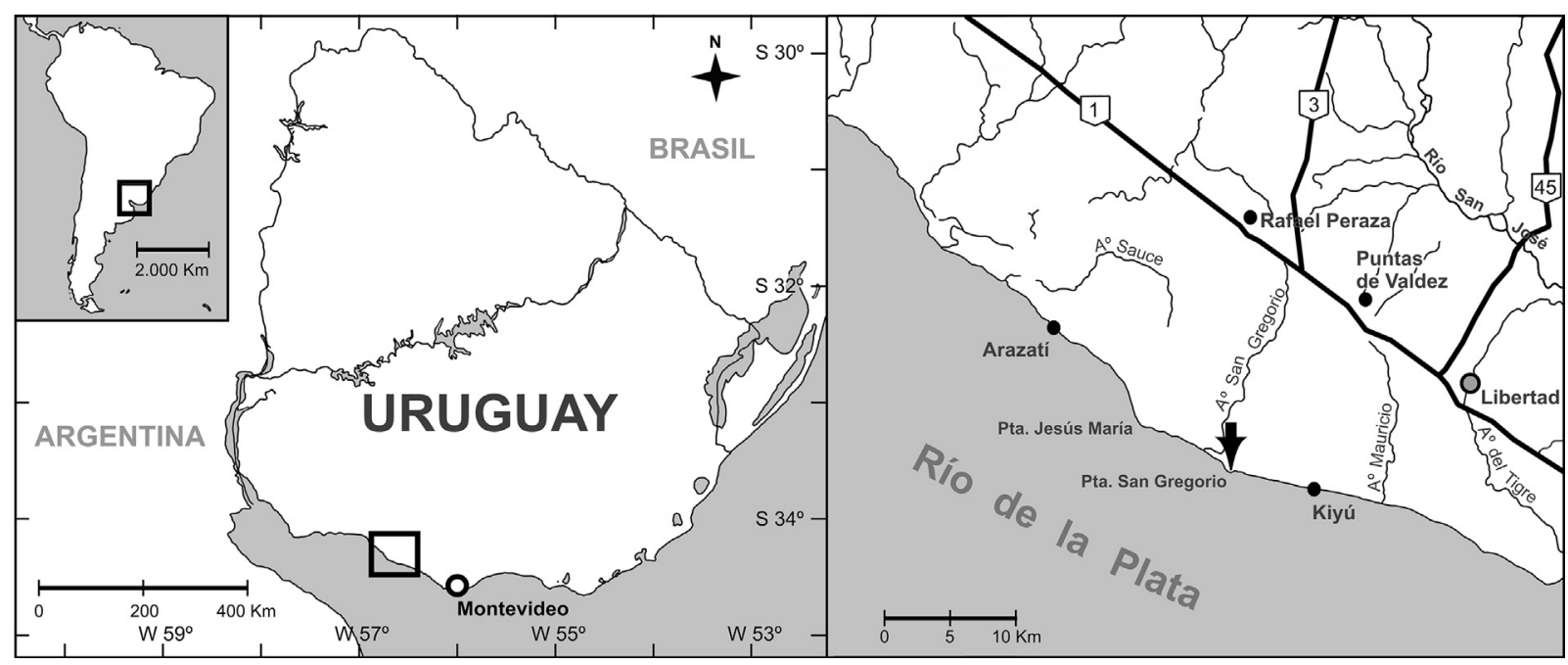

Figura 1. Mapa indicando la zona en que se recolectaron los ejemplares estudiados (FC-CVF 1825, FC-CVF 2759). La flecha señala el punto de recolecta del ejemplar FC-CVF 1825.

Nopachtus sp. (fig. 2)

\section{Nuevo material referido}

Dos osteodermos aislados correspondientes a la coraza dorsal (FC-CVF 1825 y FC-CVF 2759).

\section{FC-CVF 1825 (fig. 2, A-C)}

\section{Procedencia geográfica y estratigráfica}

Barrancas de Punta San Gregorio, balneario Kiyú. Departamento de San José, Uruguay ( $34^{\circ} 41^{\prime}$ S; $56^{\circ} 49^{\prime}$ O; fig. 1). Recolectado por uno de los autores (A.R.) en 2004, en la resaca de la playa al pie de las barrancas. Formación Camacho (Mioceno Tardío) o Formación Raigón (Mioceno Tardío-Pleistoceno Medio).

\section{Observaciones}

El ejemplar presenta un contorno pentagonal. En la cara externa posee una figura central rodeada por una hilera de figuras periféricas bien definidas. La figura central es de contorno subcircular, de superficie lisa y con uno de sus bordes levemente más elevado con respecto al nivel de las demás figuras. Las figuras periféricas que se encuentran en contacto con la figura central son 12, presentan contornos regulares pentagonales o hexagonales y conforman un anillo claramente diferenciable. Adicionalmente, se observa una segunda hilera de figuritas periféricas de menor tamaño que las de la hilera más proximal, de formas irregulares en número de 25 . El resto está conformado por algunas figuritas periféricas de distribución irregular -algunas marginales, incompletas, probablemente compartidas con osteodermos adyacentes-. En casi todas las intersecciones de los surcos se aprecian forámenes más notorios que en el otro ejemplar (FC-CVF 2759, ver más adelante). La cara interna es suavemente cóncava, de superficie lisa y con presencia de algunos forámenes de distribución irregular, los cuales son de mayor tamaño hacia la parte central de la placa.

\section{FC-CVF 2759 (fig. 2, D-F)}

\section{Procedencia geográfica y estratigráfica}

Barrancas de Punta San Gregorio, balneario Kiyú. Barrancas costeras del Departamento de San José, Uruguay ( $34^{\circ} 41^{\prime} \mathrm{S}$; $56^{\circ} 49^{\prime}$ O; fig. 1). Recolectado por Libertad Lausarot a comienzos de la década del 2000, en la resaca de la playa al pie de las barrancas. Formación Camacho (Mioceno Tardío) o Formación Raigón (Mioceno Tardío-Pleistoceno Medio).

\section{Observaciones}

El osteodermo está levemente rodado, su contorno es prácticamente cuadrangular. Al igual que en FC-CVF 1825, en vista externa presenta una figura central, con una hilera proximal de figuritas periféricas de contornos regulares en número de 12, pentagonales o hexagonales, formando un anillo definido alrededor de dicha figura. Esta última, como en el osteodermo anteriormente descrito, es de contorno subcircular y de superficie lisa, también ligeramente más elevada que el resto de las figuritas. Las restantes figuritas que se observan son más pequeñas que las que definen el anillo adyacente a la figura central, y son de formas poligonales: cuadrangulares, pentagonales y hexagonales. Se reconoce una segunda fila incompleta de estas en número de 22. Como sucede en FC-CVF 1825, presenta algunas figuritas periféricas marginales incompletas, correspondientes a hileras periféricas compartidas con osteodermos contiguos. Los forámenes que se observan en la mayor parte de las suturas del ejemplar son menos evidentes que en el anterior. La cara interna es bastante plana y, al igual que en FC-CVF 1825, su superficie es lisa y presenta algunos forámenes. 
A

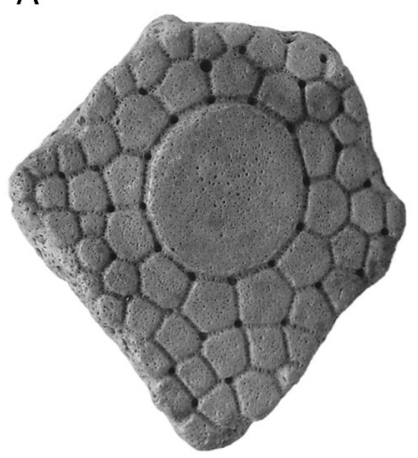

D

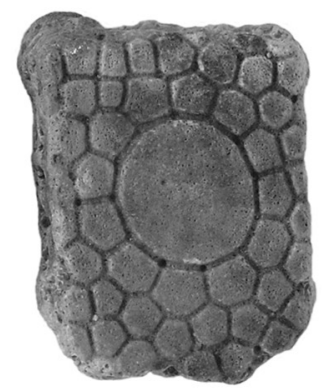

B

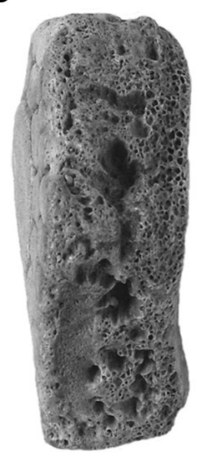

$\mathrm{E}$

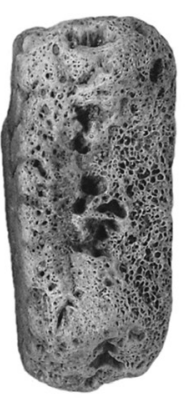

C

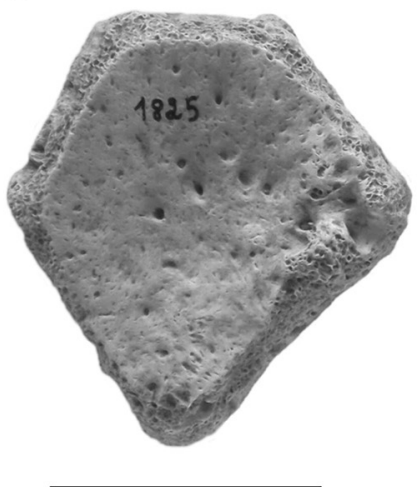

$\mathrm{F}$

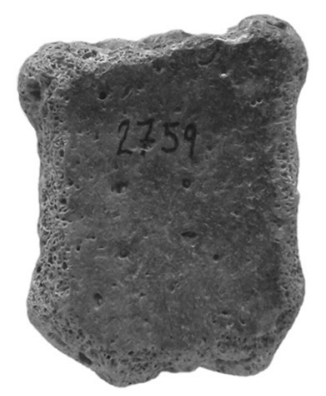

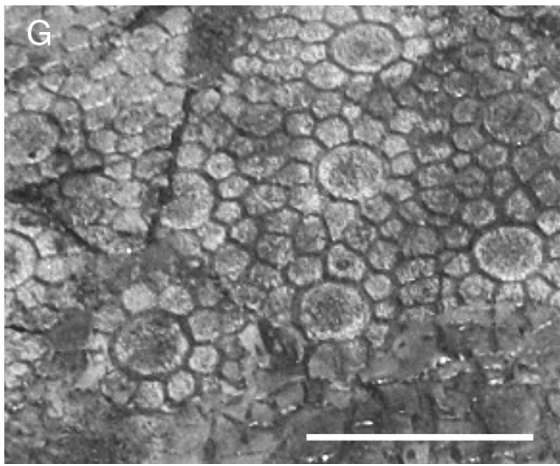

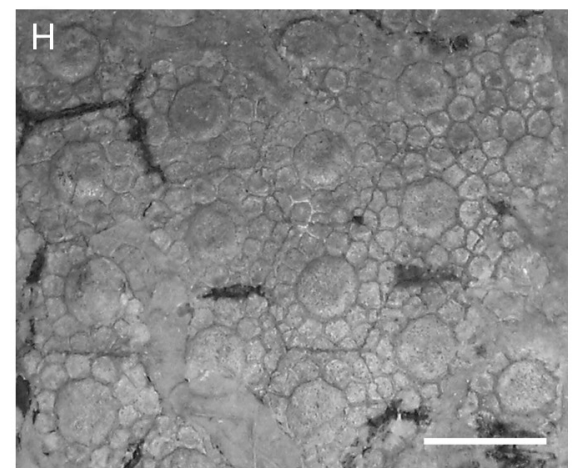

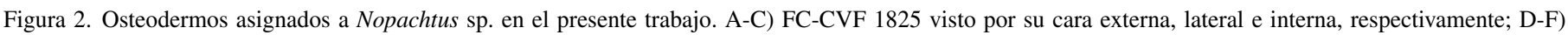

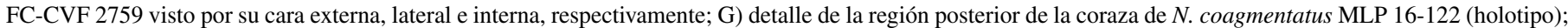
H) detalle de la región posterior de la coraza de $N$. cabrerai MACN-Pv 2670 (holotipo). Escalas $5 \mathrm{~cm}$.

\section{Discusión}

El análisis comparativo del material estudiado con taxones posiblemente afines registrados en Argentina y Uruguay arroja los siguientes resultados: una correspondencia del material con Propanochthus ( $P$. bullifer) se descarta rápidamente, dado que en este taxón la figura central de los osteodermos de la coraza se encuentra rodeada por entre 3 y 5 hileras de figuritas periféricas (Castellanos, 1942; Zamorano, 2012a; Zamorano, 2013).

En los osteodermos aquí analizados (FC-CVF 2759 y FCCVF 1825), atribuidos a Nopachtus sp., la segunda hilera, como ya se mencionó, tiene 25 y 22 figuritas, respectivamente; es decir, tienen más que en la de Pseudohoplophorus absolutus Perea (2 figuritas, solo en algunas placas de la zona lateral, ver Perea, 2005), Plohophorus figuratus Ameghino (16 a 17, según Ameghino, 1889) y Stromaphorus compressidens (Moreno y Mercerat) (con un máximo de 21, ver Cabrera, 1944, fig. 18), pero menos que en la de Phlyctaenopyga ameghini Ameghino, 1889 (que llegan a 27, ver Zamorano et al., 2011). Por otra parte, de las 6 especies que integran el género Panochthus, únicamente $P$. intermedius presenta figuras centrales en osteodermos de buena parte de la coraza dorsal. No obstante, en esta última especie las figuras centrales son de superficie rugosa, y las figuritas periféricas son proporcionalmente más pequeñas y se disponen en mayor cantidad de hileras, a diferencia de lo que acontece en Nopachtus (Zamorano, 2012a). En las restantes especies de Panochthus solamente se observan figuras centrales en la zona marginal, aunque sin alcanzar un desarrollo tan notorio como en el material estudiado. No obstante, por su morfología las placas descritas no corresponden a la zona marginal, sino a la región dorsal.

Dada la notoria correspondencia en la ornamentación de las placas estudiadas con respecto a los ejemplares del género Nopachtus, y atendiendo a su vez a las comparaciones establecidas 


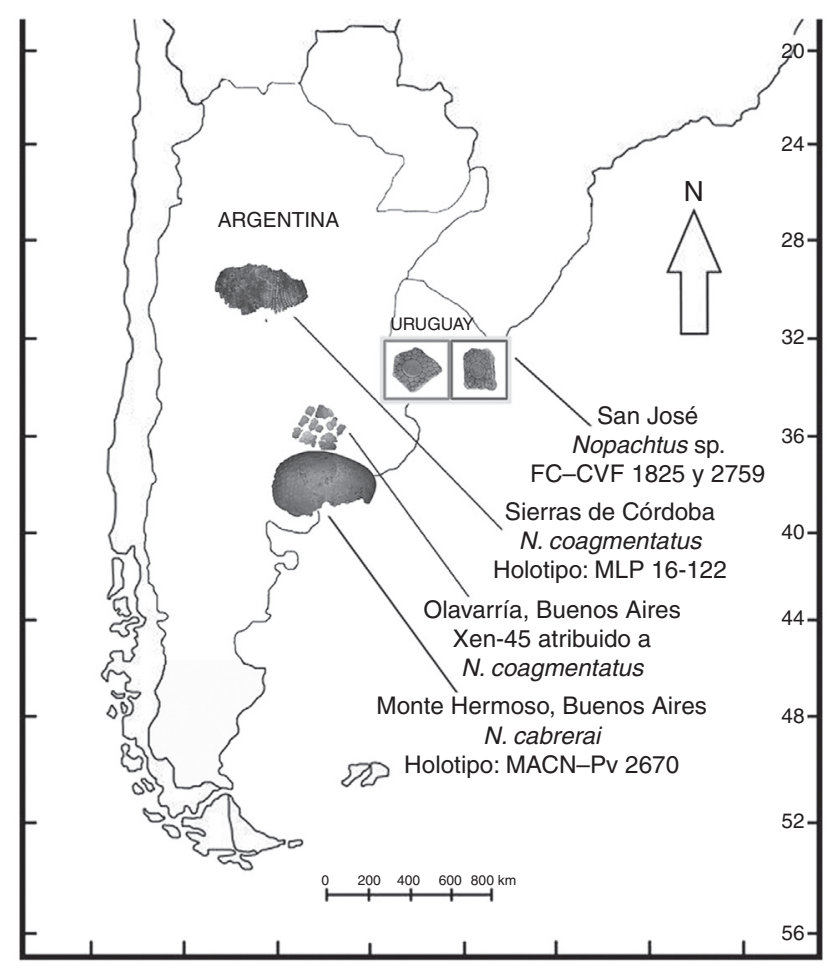

Figura 3. Distribución de los materiales de Nopachtus.

en párrafos anteriores, se considera que el material presentado reúne cualidades suficientes para ser asignado al citado género, ampliándose así la distribución geográfica de este taxón a Uruguay (fig. 3). Los osteodermos aquí descriptos no se asignan a ninguna de las 2 especies de Nopachtus, puesto que se considera que las características de los materiales aquí presentados solamente permiten su atribución a nivel genérico. Las figuras centrales, como ya se ha dicho, son ligeramente elevadas. En Nopachtus, dichas figuras son elevadas en la región posterior de la coraza dorsal, en $N$. cabrerai son de elevadas a francamente convexas, con aspecto de ampollas, en $N$. coagmentatus son mucho menos elevadas (fig. 2, G-H). A su vez, cabe señalar que, hasta el momento, en los gliptodóntidos no es posible identificar a qué lado de la coraza (derecha/izquierda) pertenecen los osteodermos; esta identificación sí se puede hacer en dasipódidos (Scillato-Yané, 1975, 1982; Zamorano et al., 2015). Sobre los osteodermos presentados en esta contribución, solo se puede precisar que, teniendo en cuenta que las figuras centrales son levemente más elevadas que el resto de las figuritas periféricas y que de estas últimas hay 2 hileras completas que rodean la figuras central, estos osteodermos (FC-CVF 1825 y FC-CVF 2759) corresponden a la región central o posterior de la coraza dorsal (Zamorano, 2012a; Zamorano et al., 2011).

En cuanto a la posible procedencia estratigráfica del material estudiado, actualmente se reconocen 2 unidades litoestratigráficas para las barrancas en la zona de los hallazgos (Bossi, Ortiz y Perea, 2009): la formación Camacho (Mioceno Tardío) de origen marino y costero, y la formación Raigón (Mioceno Tardío-Pleistoceno Medio) de origen continental fluvial. La primera de ellas ha arrojado restos de varios mamíferos de afinidad Huayqueriense, incluyendo xenartros, roedores cardiaterinos, notoungulados y litopternos; así como otros de afinidades Laventense, Montehermosense, Chapadmalalense y Marplatense (Perea, Rinderknecht, Ubilla, Bostelman y Martínez, 2013). Por su parte, el registro de la formación Raigón abarca un amplio lapso temporal, incluyendo taxones de afinidades montehermosenses, tales como el notoungulado Trigodon aff. T. gaudryi y el tardígrado Proscelidodon patrius Ameghino (Mones, 1967; Perea et al., 2013). Y también ensenadensesbonaerenses, como Catonyx tarijensis (Ameghino) (McDonald y Perea, 2002; Perea et al., 2013). De acuerdo con estos antecedentes, se estima que el material asignado a Nopachtus en la presente contribución puede corresponder a cualquiera de las 2 unidades. Futuros hallazgos permitirán establecer consideraciones taxonómicas y estratigráficas más precisas en relación con la presencia del género en el país.

El estudio de estos osteodermos de la coraza dorsal, perfectamente diagnósticos, asignables a Nopachtus sp., proporcionan por primera vez el registro del género para Uruguay, y este nuevo registro es el más oriental del género. Este gliptodóntido, hasta ahora era exclusivo de Argentina, incrementa la biodiversidad de megafauna de Uruguay.

\section{Agradecimientos}

A la Prof. Libertad Lausarot por la donación de uno de los ejemplares estudiados. Contribución al proyecto ANII FCE_1_2011_1_6752 Mamíferos xenartros, estratigrafía, cronología y ambientes del Cenozoico de Uruguay (D.P.). A Gustavo J. Scillato-Yané por recomendaciones sobre el manuscrito; así como a los revisores, por enriquecer el trabajo.

\section{Referencias}

Ameghino, F. (1888). Rápidas diagnosis de algunos mamíferos fósiles nuevos de la República Argentina. Buenos Aires: Pablo E. Coni e hijos.

Ameghino, F. (1889). Contribución al conocimiento de los mamíferos fósiles de la República Argentina. Actas de la Academia Nacional de Ciencias en Córdoba, 6. I-XCVIII.

Bossi, J., Ortiz, A. y Perea, D. (2009). Pliocene to middle Pleistocene in Uruguay: A model of climate evolution. Quaternary International, 210, 37-43.

Cabrera, A. (1944). Los gliptodontoideos del Araucaniano de Catamarca. Revista del Museo de La Plata (nueva serie). Paleontología, 3, 5-76.

Castellanos, A. (1925). Breves notas sobre evolución de la coraza y del extremo caudal en los Loricata (=Hicanodonta). Revista de El Círculo, número extraordinario, 93-96.

Castellanos, A. (1927). Descripción de un fragmento de tubo caudal de un nuevo dedicurino en relación con sus géneros afines. Anales del Museo de Historia Natural de Montevideo, 2, 263-300.

Castellanos, A. (1942). A propósito de los géneros Plohophorus, Nopachthus y Panochthus (Tercera parte). Publicaciones de la Facultad de Ciencias Matemáticas, Físico-Químicas y Naturales Aplicadas a la Industria, Universidad Nacional del Litoral. Serie Técnico-Científica, 21, 417-592.

Fernicola, J. C. (2008). Nuevos aportes para la sistemática de los Glyptodontia Ameghino, 1889 (Mammalia, Xenarthra, Cingulata). Ameghiniana, 45, 553-574.

Fernicola, J. C. y Porpino, K. O. (2012). Exoskeleton and systematics: a historical problem in the classification of Glyptodonts. Journal of Mammalian Evolution, 19, 171-183.

Hoffstetter, R. (1958). Edentata: Xenarthra. En J. Piveteau (Dir.), Traité de Paleontologie. Tome 6. Vol. 2. Paris: Masson et Cie, Éditeurs. 
McDonald, H. G. y Perea, D. (2002). The large scelidothere Catonyx tarijensis (Xenarthra, Mylodontidae) from the Pleistocene of Uruguay. Journal of Vertebrate Paleontology, 22, 677-683.

Mones, A. (1967). Notas paleontológicas uruguayas, 1. Trigodon Amegh., 1882 (Toxodonta, Notoungulata) en la fauna pliocena superior de las barrancas de San Gregorio, Dpto. de San José, Uruguay. Comunicaciones Zoológicas del Museo de Historia Natural de Montevideo, 9, 1-4.

Mones, A. (1986). Palaeovertebrata sudamericana. Catálogo sistemático de los vertebrados fósiles de América del Sur. Parte I. Lista preliminar y bibliográfica. Courier Forschungsinstitut Senckenberg, 82, $1-625$.

Moreno, F. P. (1888). Informe preliminar de los progresos del Museo La Plata durante el primer semestre de 1888, presentado al señor ministro de Obras Públicas de la provincia de Buenos Aires. Boletín del Museo La Plata, 2, $1-35$.

Paula-Couto, C. de. (1979). Tratado de Paleomastozoologia. Rio de Janeiro: Academia Brasileira de Ciências.

Perea, D. A. (2005). Pseudoplohophorus absolutus n. sp. (Xenarthra, Glyptodontidae), variabilidad en Sclerocalyptinae y redefinición de una biozona del Mioceno superior de Uruguay. Ameghiniana, 42, 175-190.

Perea, D., Rinderknecht, A., Ubilla, M., Bostelman, E. y Martínez, S. (2013). Mamíferos y estratigrafía del Neógeno en Uruguay. Publicación especial 14. En D. Brandoni y J. I. Noriega (Eds.), El Neógeno de la Mesopotamia argentina (pp. 186-200). Buenos Aires: Asociación Paleontológica Argentina.

Porpino, K. O., Fernicola, J. C. y Bergqvist, L. (2010). Revisiting the intertropical brazilian species Hoplophorus euphractus (Cingulata, Glyptodontoidea) and the phylogenetic affinities of Hoplophorus. Journal of Vertebrate Paleontology, 30, 911-927.

Scillato-Yané, G. J. (1975). Nuevo género de Dasypodidae (Edentata, Xenarthra) del Plioceno de Catamarca (Argentina). Algunas consideraciones filogenéticas y zoogeográficas sobre los Euphractini. Actas del I Congreso Argentino de Paleontología y Bioestratigrafía, 2, 449-461.

Scillato-Yané, G. J. (1982). Los Dasypodidae (Mammalia, Edentata) del Plioceno y Pleistoceno de Argentina. Tesis doctoral. Facultad de Ciencias Naturales y Museo, Universidad Nacional de La Plata.

Scillato-Yané, G. J., Carlini, A. A., Vizcaíno, S. F., Ortiz-Jaureguizar, E., \& Los Xenartros. (1995). In M. T. Alberdi, G. Leone, y E. P. Tonni (Eds.), Evolución biológica y climática de la región Pampeana durante los últimos cinco millones de años. Un ensayo de correlación con el Mediterráneo occidental (pp. 181-209). Madrid: Monografías. Museo Nacional de Ciencias Naturales, Consejo Superior de Investigaciones Científicas.

Simpson, G. G. (1945). The principles of classification and a classification of mammals. Bulletin of the American Museum of Natural History, 85, 1-350.

Toriño, P. y Perea, D. (2008). Tertiary glyptodontids from Uruguay. II: the Hoplophorinae case and final considerations. Libro de resúmenes: 251. Neuquén: III Congreso Latinoamericano de Paleontología de Vertebrados.

Toriño, P. y Rinderknecht, A. (2005). Nuevo registro de Panochthini (Mammalia: Glyptodontidae) en el departamento de San José. Montevideo: Actas de las VIII Jornadas de Zoología del Uruguay. II Encuentro de Ecología del Uruguay.

Zamorano, M. (2012a). Los Panochthini (Xenarthra, Glyptodontidae): sistemática y evolución. Tesis doctoral. Facultad de Ciencias Naturales y Museo, Universidad Nacional de La Plata.

Zamorano, M. (2012b). Los Panochthini (Xenarthra, Glyptodontidae): sistemática y evolución. Mastozoología Neotropical, 19, 382-384.

Zamorano, M. (2013). Diagnosis y nueva descripción de Propanochthus bullifer (Burmaister) (Xenarthra, Glyptodontidae). Consideraciones bioestratigráficas y cronológicas de su procedencia. Spanish Journal of Palaeontology, 28, 283-292.

Zamorano, M. y Brandoni, D. (2013). Phylogenetic analysis of the Panochthini (Xenarthra, Glyptodontidae), with remarks on their temporal distribution. Alcheringa, 37, 442-451.

Zamorano, M., de los Reyes, M., Poiré, D. y Scillato-Yané, G. J. (2015). Primer registro de Nopachtus coagmentatus (Glyptodontidae, «Hoplophorinae», «Panochthini») en la región Pampeana, Argentina. Madrid: Estudios Geológicos.

Zamorano, M., Scillato-Yané, G. J., González-Ruíz, L. R. y Zurita, A. E. (2011). Revisión de los géneros Nopachtus Ameghino y Phlyctaenopyga Cabrera (Xenarthra, Glyptodontidae, Hoplophorinae) del Mioceno Tardío y Plioceno de Argentina. Revista del Museo Argentino de Ciencias Naturales (nueva serie), 13, 59-68.

Zamorano, M., Scillato-Yané, G. J. y Zurita, A. E. (2014). Revisión del género Panochthus Glyptodontidae, «Hoplophorinae», «Panochthini»). Nuevas diagnosis y descripciones de sus especies. Revista del Museo de La Plata, 14, 1-46.

Zamorano, M., Zurita, A. E. y Scillato-Yané, G. J. (2013). Contribution to the systematic of some Glyptodontidae (Xenarthra, Cingulata). La Rioja, Argentina: XXVII Jornadas Argentinas de Paleontología de Vertebrados (La Rioja), Libro de resúmenes: 99 